\title{
OCDMA/OCDMA Overloading Scheme for Cellular DS-CDMA Using Orthogonal Gold Codes and Complex Scrambling
}

\author{
Preetam KUMAR, Saswat CHAKRABARTI \\ G S Sanyal School of Telecommunications, IIT Kharagpur, India \\ Email:preetam@gssst.iitkgp.ernet.in,saswat@ece.iitkgp.ernet.in \\ Received on May 5, 2008; revised and accepted on June 27, 2008
}

\begin{abstract}
Overloading is a method to extend capacity limitation of multiple access techniques. The system becomes overloaded, when the number of users exceeds the signal dimensions. One of the efficient schemes to overload a CDMA system is to use two sets of orthogonal signal waveforms $(\mathrm{O} / \mathrm{O})$. In this paper, the BER performance of a new overloading scheme using scrambled orthogonal Gold code (OG/OG) sets is evaluated with soft decision interference cancellation (SDIC) receiver. When complex scrambling is not used, it is shown that OG/OG scheme provides 25\% (16 extra users) channel overloading for synchronous DS-CDMA system in an AWGN channel, with an SNR degradation of about $0.35 \mathrm{~dB}$ as compared to single user bound at a BER of 1e-5. We have evaluated the overloading performance, when two set are scrambled with set specific deterministic or random complex scrambling sequence. It is shown that the amount of overloading increases significantly from $25 \%$ to $63 \%$ (40 extra users) by using random complex scrambling for N=64. For deterministic (periodic) scrambling, the overloading percentage increases considerably to 78 . On a Rayleigh fading channel, an overloading of $40 \%$ is obtained without scrambling at a BER of 5e-4 with near single user performance. With complex scrambling overloading $\%$ increases considerably to $100 \%$.
\end{abstract}

Keywords: DS-CDMA, Orthogonal Codes, Overloading, Interfernce Cancellation

\section{Introduction}

Efficient use of the available radio spectrum is an important requirement for future wireless communication. The number of users supported in a DS-CDMA cellular system is typically less than spreading factor $(\mathrm{N})$, and the system is said to be underloaded. As the demand for cellular CDMA increases, the number of users naturally exceeds the available dimension due to bandwidth limitation. Overloading is a technique to accommodate more number of users than the spreading factor $\mathrm{N}$. This is an efficient scheme to increase users in a fixed bandwidth which is of practical interest to mobile system operators. But the increase in capacity is obtained with a cost in BER and receiver complexity. In fact this type of channel overloading is provisioned in the $3 \mathrm{G}$ standard [1].

Among the approaches described in the literature, the most efficient ones use multiple sets of orthogonal codes [2]. The concept of overloading in a DS-CDMA system using two sets of orthogonal codes is explained with the help of Figure1. For the first $\mathrm{N}$ users, the system allocates orthogonal codes drawn from the first set of $\mathrm{N}$ codes. When the number of intending users exceeds ' $\mathrm{N}$ ', the excess users are accommodated in the system by providing suitable codes drawn from a second set of $\mathbf{M}$ codes. In this way, we are able to accommodate greater number of users $(K)$ than the spreading length $N(K>N)$, and the cell becomes overloaded.

The number of active users $(\mathrm{K})$ in a conventional synchronous orthogonal CDMA environment is limited by the spreading factor N, which is WT where $\mathrm{W}$ is the transmission bandwidth and $\mathrm{T}$ is the duration of a symbol. When $\mathrm{K}$ exceeds $\mathrm{N}$, the system becomes overloaded and the signatures are no longer orthogonal. This leads to multiple access interference (MAI). In an overloaded system, a conventional matched filter receiver is not optimal, due to the high level of MAI. Multiuser detection (MUD) is required in order to obtain a 
satisfactory performance of the users. Linear MUDs, such as the decorrelator, the minimum mean squared error detector or linear decision directed interference cancellation are devised to detect users in an underloaded system. The Maximum Likelihood (ML) detection is not an option because of its complexity that is exponential in the number of users. The nonlinear MUDs such as multistage parallel interference cancellation (PIC) and successive interference cancellation (SIC) [3], have good complexity- performance trade-off as compared to other MUDs. Hence these MUDs are suitable for overloaded systems.

It is interesting to note that several studies have been made in the recent past to understand, analyze and evaluate the detrimental effects of overloading. Almost all studies consider the uplink or reverse link and several studies suggest usage of appropriate multiuser detection (MUD) schemes at the base station receiver. For example, a method of accommodating $\mathrm{K}=N+M$ users in an $\mathrm{N}$ dimensional signal space that does not compromise the minimum Euclidean distance of the orthogonal signaling has been presented in [4] for AWGN channel. A tree-like correlation coefficient structure of user signatures suitable for optimal multiuser detection has been proposed in [5]. In another approach, two sets of orthogonal codes which are orthogonal within the sets is introduced in [6]. In [6], the orthogonal sets are generated using Walsh-Hadamard (WH) codes, where the same $\mathrm{WH}$ code set is scrambled with set specific scrambling sequence $(\mathrm{s}-\mathrm{O} / \mathrm{O})$. An iterative multistage detection technique has been proposed to cancel the interference between the two sets of user. In [7], it is shown that for uncoded BPSK modulated CDMA signal with $\mathrm{N}=64$, an overloading of $11 \%$ can be achieved in an AWGN channel for s-O/O scheme. Another kind of receiver simplification is presented in [8], where signals are divided into groups that are orthogonal to each other. A new overloading scheme using hybrid techniques has been proposed in [9], where the spreading codes and transmission modes are different for the two sets to increase the overloading performance. The attractive property of the overloading scheme was the incentive to integrate a particular type of $\mathrm{O} / \mathrm{O}$, called quasisynchronous sequences (QOS) [10], into cdma2000 standard [11].

To the best of our knowledge, the usage of orthogonal Gold codes has not been considered in any of the overloading schemes. In [12], a new method for generating different orthogonal sets of same length has been proposed. The new algorithm generates $(N-1)$ distinct, orthogonal sets of $N$ sequences of length $N$. It has been shown that the peak value of crosscorrelation between different sets of same length is less than half the sequence length for $N \geq 32$. Such sequence sets would offer low intracell interference, when used in overloaded environment. Recently, the present authors have proposed a new overloading scheme using a set of Gold codes [13], which provides better performance than s-

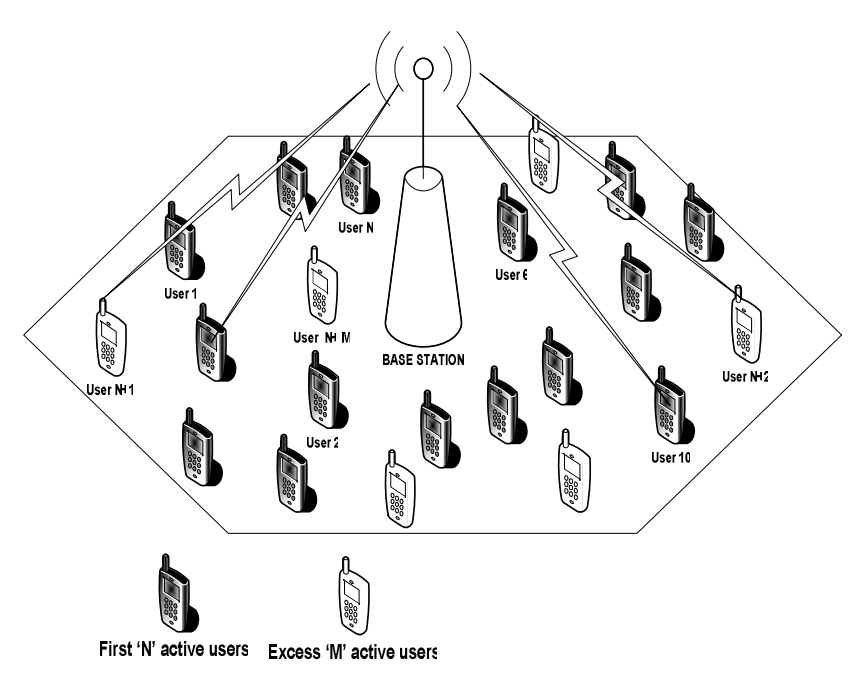

Figure 1. Overloading scheme in a DS-CDMA cellular system.

O/O scheme [7]. In this paper, we have evaluated the BER performance using orthogonal Gold code (OG/OG) sets with IMSD schemes. An efficient iterative multistage detection with soft decision interference cancellation is used to increase the amount of overloading.

This paper is organized as follows. In the next section, we describe the system model for the O/O overloading scheme. In section 3 we explain the IMSD operation and describe the process of iterative interference cancellation. Simulation results are presented and discussed in Section 4. Finally, we present the conclusion of this paper.

\section{System Model for OCDMA/OCDMA}

In the section we consider a DS-CDMA system with processing gain $N$ and the number of users $K$ users $(=M+N)$, where $M$ is the number of set- 2 users. We assume that the channel is a nondispersive additive white Gaussian noise (AWGN) channel and that the different user signals are in perfect time synchronization. The discrete-time matrix model of the received BPSK modulated CDMA signal after demodulation and chip sampling may now be expressed as:

$$
r=r_{1}+r_{2}=b_{1} A_{1} S_{1}+b_{2} A_{2} S_{2}+n
$$

Here $r_{1}$ and $r_{2}$ are the received samples from set-1 and set-2 uers respectively; $\mathbf{b}_{1}=\left\{b_{11}, b_{12}, \ldots \ldots ., b_{1 N}\right\}$ and $\mathbf{b}_{2}=\left\{b_{21}, b_{22}, \ldots \ldots, b_{2 M}\right\}$ represent information bits of two sets of users, where $b_{i j} \in\{ \pm 1\} . \mathbf{S}_{1}=\left\{\mathbf{s}_{11}^{T}, \ldots \ldots . \mathbf{s}_{1 N}^{T}\right\}^{T}$ is the set of orthogonal sequences for set-1 users and is of dimension $(N \times N) . \mathbf{S}_{2}=\left\{\mathbf{s}_{21}^{T}, \ldots ., \mathbf{s}_{2 M}^{T}\right\}^{T}$ is another set of orthogonal sequences for set-2 users and is of dimension $(M \times N)$. Additive noise $\mathbf{n}$ is normally distributed with zero mean and variance equal to $\sigma^{2} . \mathbf{A}_{1}$ and $\mathbf{A}_{2}$ are the 


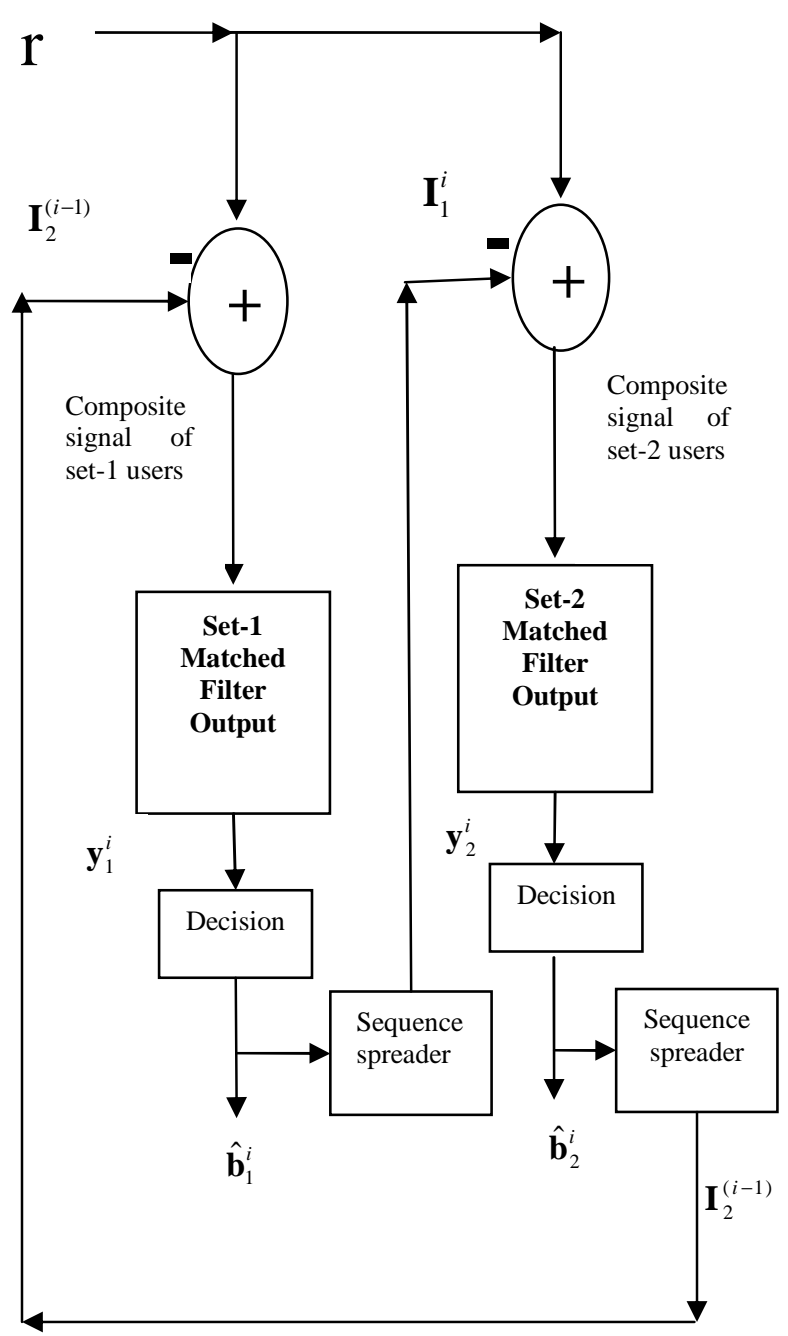

Figure 2. Block diagram of iterative multistage detection (IMSD) for overloaded DS-CDMA scheme.

diagonal matrices of received signal amplitudes of set-1 and set- 2 users respectively.

The received chip sampled and demodulated signal (1) is despread and integrated over a bit duration to get a soft estimate about a transmitted bit. These soft estimates are fed to an iterative multistage detector (IMSD) (Figure 2). As $\mathrm{N}>\mathrm{M}$ set-1 users matched filter outputs are more reliable (due to less MAI) as compared to the set-2 matched filter outputs $(\mathrm{MAI}=1)$. In the first iteration we will assume $\mathbf{I}_{2}^{0}=0$ and accordingly find $\hat{\mathbf{b}}_{1}^{1}$ and estimate $\mathbf{I}_{1}^{1}$. This estimated interference is removed from the received signal, before taking decisions on set- 2 users. Subsequently we find $\hat{\mathbf{b}}_{2}^{1}$ and estimate the interference $\mathbf{I}_{2}^{1}$ on set- 1 users from set- 2 users. In the second iteration, we obtain the refined data estimates of set- 1 users, $\hat{\mathbf{b}}_{1}^{2}$ after removing the estimated interference $\mathbf{I}_{2}^{1}$ as shown in. As we have more reliable data estimates of set- 1 users, a refined estimate of $\mathbf{I}_{1}^{2}$ is obtained in second iteration. This process repeats for required number of iterations, so that near single user performance is obtained.

When scrambling is used, the orthogonal Gold codes of both the sets are overlaid by a set-specific pseudonoise (PN) sequence which is the same for all users within the set. In other words, we have $\mathbf{S}_{1}=\frac{1}{\sqrt{N}}\left[\boldsymbol{\alpha}_{1} \boldsymbol{\alpha}_{2} \ldots \ldots . \boldsymbol{\alpha}_{N}\right]$ and $\mathbf{S}_{2}=\frac{1}{\sqrt{N}}\left[\boldsymbol{\beta}_{1} \boldsymbol{\beta}_{2} \ldots \ldots . . \boldsymbol{\beta}_{\mathrm{M}}\right]$. Let $\mathbf{P}_{1}=\left(p_{11}, p_{22}, \ldots . . p_{N 1}\right)^{T}$ and $\mathbf{P}_{2}=\left(p_{12}, p_{22}, \ldots . . p_{N 2}\right)^{T}$ designate the PN sequences overlaying the orthogonal Gold sequences in the two sets of users. In order to split the interference power evenly over the in-phase and quadrature components of the useful signal (irrespective of the carrier phase), we consider complex valued PN sequences: the chips $p_{n u}$ takes their values from the set

$$
\begin{aligned}
& C= \\
& \{\exp (j \pi / 4), \exp (j 3 \pi / 4), \exp (j 5 \pi / 4), \exp (j 7 \pi / 4)\}
\end{aligned}
$$

The scrambling sequence can be deterministic (periodic) or random. In periodic scrambling, the scrambling sequence randomly takes values form the set $C$ and it is kept constant for all symbols. On the other hand, in random complex scrambling, it takes random complex values from the set $C$ for each transmitted symbol.

In the next section, we explain iterative multistage detection scheme, which reduces the high level of interference due to overloading.

\section{Iterative Multistage Detection}

The received demodulated and chip sampled signal (1) is despread and we obtain soft outputs of the transmitted bits corrupted by multiple access interference (MAI) from other users and AWGN noise. In conventional matched filter detection, these outputs are fed to the decision device to make hard decisions of the transmitted information bits. In this paper, iterative multistage detection (IMSD) technique is used to remove the MAI between two sets users. The basic principle of this receiver is to iteratively remove the estimated interference from each set due to the users of other set in multiple stages such that near single user performance is achieved. The interference power from set2-user (assuming that the useful signal power is normalized) is $1 / \mathrm{N}$, and therefore the total interference power that affects set1-users is $\mathrm{M} / \mathrm{N}$. As long as $\mathrm{M}$ remains small compared to $\mathrm{N}$, preliminary decisions can be made on the symbols transmitted by set1-users with some good reliability. But each of the set2-users gets an interference power of $\mathrm{N}(1 / \mathrm{N})=1$ from set1-users. Clearly the bit error (BER) performance will be poor for this set of users if detection is made prior to interference cancellation. As set1-users are detected with some good reliability, we can 
estimate the interference created from this set on set2users. This estimated interference is removed from set2uers before making the decision. Now in second iteration, interference from set2-users on set-1 are estimated form the first iteration outputs of set- 1 and a more reliable set1 bits are obtained. This process continues till we get a near single user performance.

To explain the operation of IMSD the following notations are used: $\hat{\mathbf{b}}_{1}^{i}$ and $\hat{\mathbf{b}}_{2}^{i}$ are decisions about set-1 and set- 2 user data bits at the $i$-th iteration; $\mathbf{y}_{1}^{i}$ and $\mathbf{y}_{2}^{i}$ are set- 1 and set- 2 matched filter outputs at $i$-th iteration respectively.

At each stage of iteration, the decision on an information bit is made according to the following expressions:

$$
\begin{gathered}
\hat{\mathbf{b}}_{1}^{i}=\bar{\phi}\left(\mathbf{y}_{1}^{\mathrm{i}}\right)=\bar{\phi}\left(\mathbf{S}_{1}^{T}\left(\mathbf{r}-\mathbf{I}_{2}^{(i-1)}\right)\right) \\
\hat{\mathbf{b}}_{2}^{i}=\bar{\phi}\left(\mathbf{y}_{2}^{i}\right)=\bar{\phi}\left(\mathbf{S}_{2}^{T}\left(\mathbf{r}-\mathbf{I}_{1}^{i}\right)\right)
\end{gathered}
$$

Here the reconstructed interference for two groups in $i$ th iteration are $\mathbf{I}_{1}^{i}=\hat{\mathbf{b}}_{1}^{i} \mathbf{A}_{1} \mathbf{S}_{1}$ and $\mathbf{I}_{2}^{i}=\hat{\mathbf{b}}_{2}^{i} \mathbf{A}_{2} \mathbf{S}_{2}$. We assume that the reconstructed interference for the first group of users in the first iteration is zero i.e., $\mathbf{I}_{2}^{0}=0$. Matched filter outputs after interference cancellation form the decision vectors $\mathbf{y}_{1}^{i}$ and $\mathbf{y}_{2}^{i}$ for set- 1 and set- 2 user data bits respectively.

We have assumed equal power, equal phase synchronous users in a single cell environment over an AWGN channel. The set-1 matched filters outputs in matrix form may be expressed as

$$
\begin{gathered}
\mathbf{y}_{1}^{i}=\mathbf{S}_{1}^{T}\left(\mathbf{r}-\mathbf{I}_{2}^{(i-1)}\right) \\
\mathbf{y}_{\mathbf{1}}^{\mathbf{i}}=\mathbf{S}_{1}^{T}\left(\mathbf{b}_{\mathbf{1}} \mathbf{A}_{1} \mathbf{S}_{1}+\mathbf{b}_{2} \mathbf{A}_{2} \mathbf{S}_{2}+\mathbf{n}-\hat{\mathbf{b}}_{2}^{(i-1)} \mathbf{A}_{2} \mathbf{S}_{2}\right) \\
=\mathbf{b}_{\mathbf{1}}+\mathbf{S}_{1}^{T} \mathbf{S}_{2} \mathbf{A}_{2}\left(\mathbf{b}_{2}-\hat{\mathbf{b}}_{2}^{(i-1)}\right)+\mathbf{S}_{1}^{T} \mathbf{n}
\end{gathered}
$$

In the case of AWGN channel, amplitude matrix is an identity matrix, i.e., $\mathbf{A}=\mathbf{I}$. For the $l$-th user of set-1, the matched filter output during $\mathrm{i}$-th iteration is

$$
y_{1, l}^{i}=b_{1, l}+\sum_{\mathrm{k}=1}^{\mathrm{M}}\left(b_{2, k}-\hat{b}_{2, k}^{(i-1)}\right) \rho_{l, k}+z_{l}
$$

where $l=1,2,3 \ldots \ldots \ldots, \mathrm{N}$ and $z_{l}=\left[\mathrm{S}_{1}^{T} \mathrm{n}\right]_{l}$ is the noise sample for $l$-th user. The following notations have been used: $\mathbf{s}_{1, l}=l$-th user (set-1 ) signature; $b_{1, l}=l$-th user transmitted data-bit (set-1); $b_{2, k}=k$-th user transmitted data-bit (set-2); $\hat{b}_{2, k}^{(i-1)}=k$-th user tentative decision (set2) on $b_{2, k}$ at $(i-1)^{\text {th }}$ iteration; $\rho_{l, k}=$ Normalized crosscorrelation value between set- 1 -th user and set- $k$-th user signatures. The matched filter output $y_{1, l}^{i}$ has three components:

$$
y_{1, l}^{i}=\underbrace{b_{1, l}}_{\begin{array}{l}
\text { desired } \\
\text { data }
\end{array}}+\underbrace{\sum_{\text {MAI from set }-2 \text { users }}^{M}\left(b_{2, k} \hat{-b}_{2, k}^{(i-1)}\right) \rho_{l, k}}_{\text {Total Noise }}+\underbrace{z_{l}}_{\text {Noise sample }}
$$

Considering all $\mathrm{N}$ set-1 users and sufficient number of set-2 users, we assume Gaussian approximation of MAI. So, the following expression from (7) is used to indicate the total noise:

$$
\text { Total Noise }=\sum_{k=1}^{M}\left(b_{2, k}-\hat{b}_{2, k}^{(i-1)}\right) \rho_{l, k}+z_{l}
$$

In equation (2) and (3), $\phi\left(b_{1, l}\right)$ is the decision function of $l$-th user (set-1). According to the decision function $\phi\left(b_{1, l}\right)$, IMSD can be classified as hard decision interference cancellation (HDIC) or soft decision interference cancellation (SDIC). For HDIC receiver the decision function is defined as:

$$
\phi\left(b_{1, l}\right)=\operatorname{sgn}\left(b_{1, l}\right)= \begin{cases}-1 & b_{1, l}<0 \\ 1 & b_{1, l}>0\end{cases}
$$

For SDIC except for the last iteration, where we take hard decision, in other iterations several nonlinear decision functions can be used. We have used piecewise linear approximation of hyperbolic tangent and is defined as:

$$
\phi\left(b_{1, l}\right)=\operatorname{sgn}\left(b_{1, l}\right)= \begin{cases}\frac{b_{1, l}}{\theta} & \left|b_{1, l}\right|<\theta \\ \operatorname{sgn}\left(b_{1, l}\right) & \left|b_{1, l}\right| \geq \theta\end{cases}
$$

Here $\theta$ is selected to minimize the average BER.

\section{Simulation Results}

This section presents the Monte-Carlo simulation results of the proposed scheme with SDIC technique. The simulation has been carried out in MAT-LAB to evaluate the BER performance of the proposed scheme in an AWGN channel.

Relevant simulation parameters are shown in Table 1. The value of the parameter $\theta$ is found through simulation as 0.5 for SDIC and it is fixed for all iterations. For all simulations, the system performance is evaluated by means of critical overload. We define the critical overload as the maximum achievable channel overload $\beta_{\max }=\left(\mathrm{K}_{\max }-\mathrm{N}\right) / \mathrm{N}$ with interference cancellation, so that the SNR degradation for an average BER of $1 \mathrm{e}-5$ is less than $0.35 \mathrm{~dB}$ as compared to single user performance. It is a measure for the maximum acceptable channel overload, 
Table 1. Description of some parameters relevant in simulation.

\begin{tabular}{|c|c|}
\hline Parameters & Specifications \\
\hline Transmission mode & Synchronous \\
\hline Modulation/ Spreading & BPSK/BPSK \\
\hline $\begin{array}{c}\text { Spreading factor, } \\
\text { N }\end{array}$ & Orthogonal Gold codes \\
\hline $\begin{array}{c}\text { Spreading codes } \\
\text { of users }\end{array}$ & Equal \\
\hline $\begin{array}{c}\text { Power and phase } \\
\text { Type of Receiver }\end{array}$ & SDIC \\
\hline Assumptions & $\begin{array}{c}\text { Perfect chip, symbol and } \\
\text { carrier synchronization }\end{array}$ \\
\hline
\end{tabular}

so that the system performance is degraded slightly as compared to the single user performance.

To increase the amount of overloading an efficient soft decision interference cancellation receiver is used as described in Section 3. In Figure 3, BER performance of this receiver at different overloading has been shown for $\mathrm{N}=64$ at $28 \%, 25 \%$ and $22 \%$ overloadings. It is observed that $28 \%$ overloading cannot be achieved, with less than 1.0 dB SNR degradation at an average BER of 1e-5. If we reduce the overloading to $25 \%$, the $\mathrm{SNR}$ degradation is about $0.35 \mathrm{~dB}$ as shown in Figure 3 and we can ensure a BER of $10^{-5}$ for all users. So, we can obtain a critical overload of $25 \%$, when the spreading factor $\mathrm{N}$ is 64 . We have observed that the amount of critical load is only $19 \%$, when the spreading length is reduced to 32 .

The critical channel overload for $\mathrm{s}-\mathrm{O} / \mathrm{O}$ is $3 \%$ and $11 \%$ for $\mathrm{N}=32$ and 64 respectively [7] for the same set of parameters. So there is a significant improvement in critical channel overload in OG/OG scheme as compared to $\mathrm{s}-\mathrm{O} / \mathrm{O}$ scheme.

In Figure 4, the BER performance of OG/OG scheme with random complex scrambling is shown for $\mathrm{N}=32$. Here, both the sets are scrambled by a set specific complex random scrambling sequence. We observe from the figure that, with complex scrambling the amount of overloading is $31 \%$, with about $0.35 \mathrm{~dB}$ SNR degradation as compared to single user performance. In Figure 5, overloading performance with periodic scrambling is shown. It is interesting to observe that the overloading performance increases to $50 \%$ with less than $0.35 \mathrm{~dB}$ SNR degradation.

In Figure 6, the BER performance with complex random scrambling is shown for $\mathrm{N}=64$. Here we observe that we can support 40 extra users $(63 \%$ channel overloading), with less than $0.35 \mathrm{~dB}$ SNR degradation as compared to single user bound. In Figure 7, BER performance with periodic complex scrambling is shown. It is shown that with periodic scrambling, critical load increases to $78 \%$. This is a significant amount of channel overloading, which can be obtained with complex scrambling. Hence, complex scrambling increases the amount of overloading significantly in an overloaded DSCDMA system as compared to unscrambled OG/OG scheme [13].

\section{BER Performance on a Rayleigh Fading Channel}

We notice that the case of an AWGN channel is obtained by taking the received signal amplitude matrix, $\mathbf{A}=\mathbf{I}_{k}$. The Rayleigh fading channel model can be described by fading amplitudes generated according to $a_{k}=a_{k}^{(I)}+\mathrm{j} a_{k}^{(Q)}$, where $a_{k}^{(I)}$ and $a_{k}^{(Q)}$ are independent zero-mean real Gaussian distributed random variables with variance $\sigma_{a_{k}^{(I)}}^{2}=\sigma_{a_{k}^{(Q)}}^{2}=1 / 2$.

In order to compare the performance of these schemes, we define the critical overload as the maximum achievable channel overload $\beta_{\text {max }}=M_{\max } / N=\left(\mathrm{K}_{\max }-\mathrm{N}\right) / \mathrm{N}$ with interference cancellation receiver, so that the SNR degradation as compared to a single user system at an average BER of $5 \mathrm{e}-4$ is less than $1 \mathrm{~dB}$ in an AWGN channel. It has to be emphasized that the receiver does not require any kind of user sorting to yield the desired overloading performance. As a consequence, this measure guarantees that the mean BER performance remains close to that of the ideal BER curve provided that $M<M_{\max }$. It is worth noting that the BER performance in case of perfect interference cancellation is identical to the performance of a non-overloaded system where the users are orthogonal, and also to the performance of a single-user system. The BER achieved by a single-user transmitting over a Rayleigh fading channel is given by

$$
P_{e_{b}}=\frac{1}{2}\left(1-\frac{1}{\sqrt{1+N_{0} / E_{b}}}\right)
$$

In Figure 8, the BER performance of OG/OG scheme with conventional matched filter and SDIC receiver on a Rayleigh fading channel is shown. We have considered set 1 and set 6 users for simulation. Figure 7 also shows the theoretical single-user BER performance over a Rayleigh fading channel. The channel overloading is fixed at $40 \%$ (26 extra users). It can be observed that the SNR degaradation at a BER of $5 \cdot 10^{-4}$ is about $1 \mathrm{~dB}$. So, we can obatain $40 \%$ channel overloading on a Rayleigh fading channel with the SDIC receiver for $N=64$.

Figure 9 shows the BER performance of scrambled OG/OG scheme, where a single set of orthogonal Gold code is used. The amount of overloading is fixed at $100 \%$ for $\mathrm{N}=64$. It is interesting to observe that the overloading increases considerably from $40 \%$ to $100 \%$ (64 extra users). The overloading for s-O/O [6] scheme is only $75 \%$ with complex scrambling with same set of simulation parameters. When we choose two different sets of orthogonal codes and complex scrambling, the 
achievable overloading is again $100 \%$. This is a significant amount of overloading on a flat Rayleigh fading channel.

\section{Conclusions}

Overloading is an efficient scheme to increase number of users in a DS-CDMA system. In this paper, overloading performance of OCDMA/OCDMA overloading scheme is evaluated, which uses orthogonal Gold codes. It is shown that this scheme with soft decision interference cancellation (SDIC) can overload the DS-CDMA systems by $25 \%$ at BER of $1 \mathrm{e}-5$ for $\mathrm{N}=64$, with an SNR degradation of about $0.35 \mathrm{~dB}$ as compared to single user bound. The amount of overloading increases significantly from $25 \%$ to $78 \%$, with periodic complex scrambling. On a Rayleigh flat fading channel, we can obtain an overloading of $40 \%$ at a BER of $5 \mathrm{e}-4$ without complex scrambling and SDIC receiver. With complex scrambling overloading \% increases considerably to $100 \%$.

\section{References}

[1] H. Sari, F. Vanhaverbeke, and M. Moeneclaey, "Multiple access using two sets of orthogonal signal waveforms," IEEE Communications Letters, Vol. 4, No. 1, pp. 4-6, January 2000

[2] P. Kumar, M. Ramesh, and S. Chakrabarti, "Overloading cellular DS-CDMA: A bandwidth efficient scheme for capacity enhancement," Springer-Verlag LNCS, Vol. 4904, pp.515-527, January 2008.

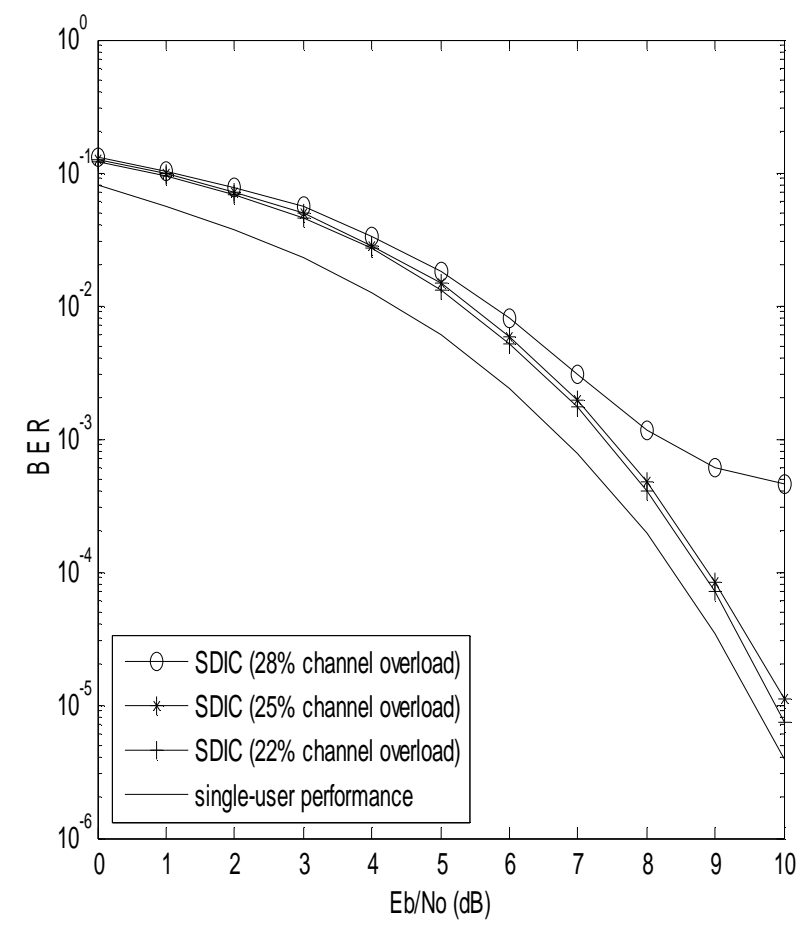

Figure 3. BER performance comparison with Soft decision interference cancellation (SDIC) with $\mathrm{N}=64$ at different values of overloading.

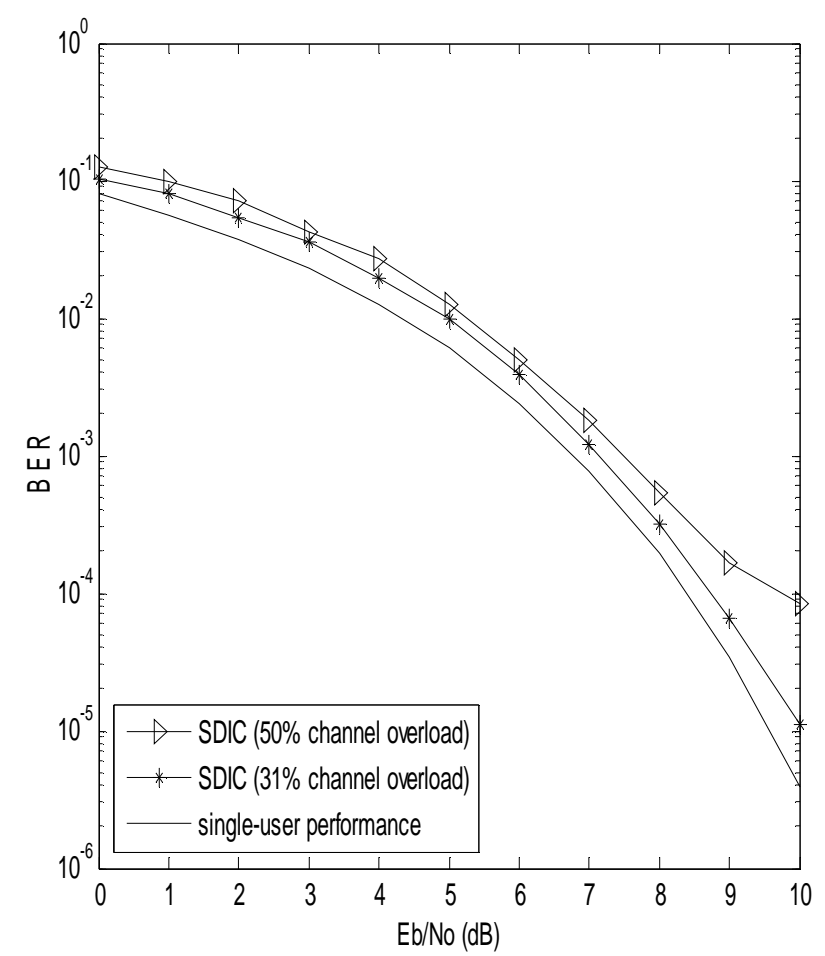

Figure 4. BER performance comparison of OG/OG scheme with random complex scrambling with SDIC recevier for $\mathrm{N}$ $=32$ at different overloadings.

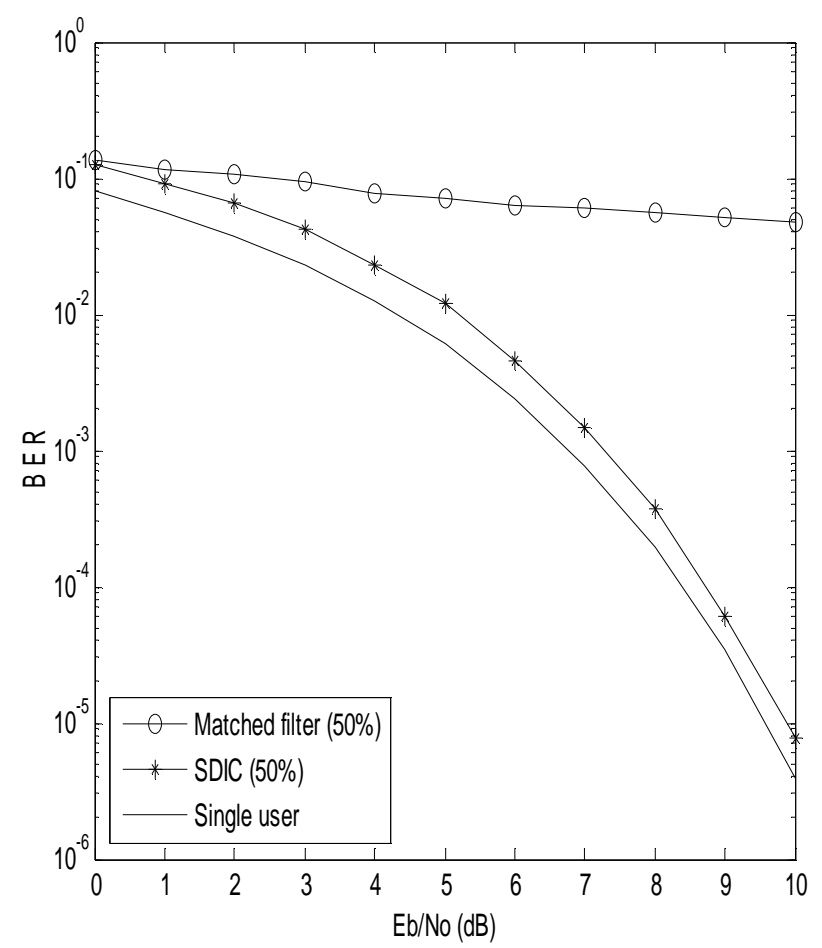

Figure 5. BER performance comparison of OG/OG scheme with periodic complex scrambling with SDIC receiver for $\mathbf{N}$ $=32$. 


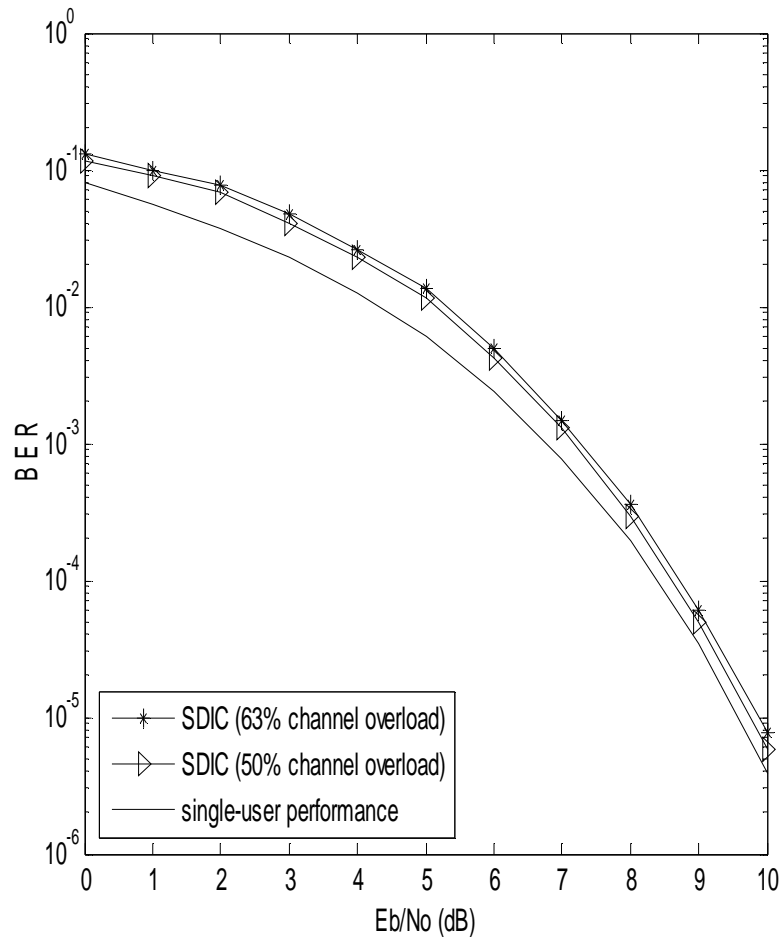

Figure 6. BER performance comparison of OG/OG scheme with random complex scrambling with Soft decision Interference cancellation (SDIC) for $\mathbf{N}=64$.

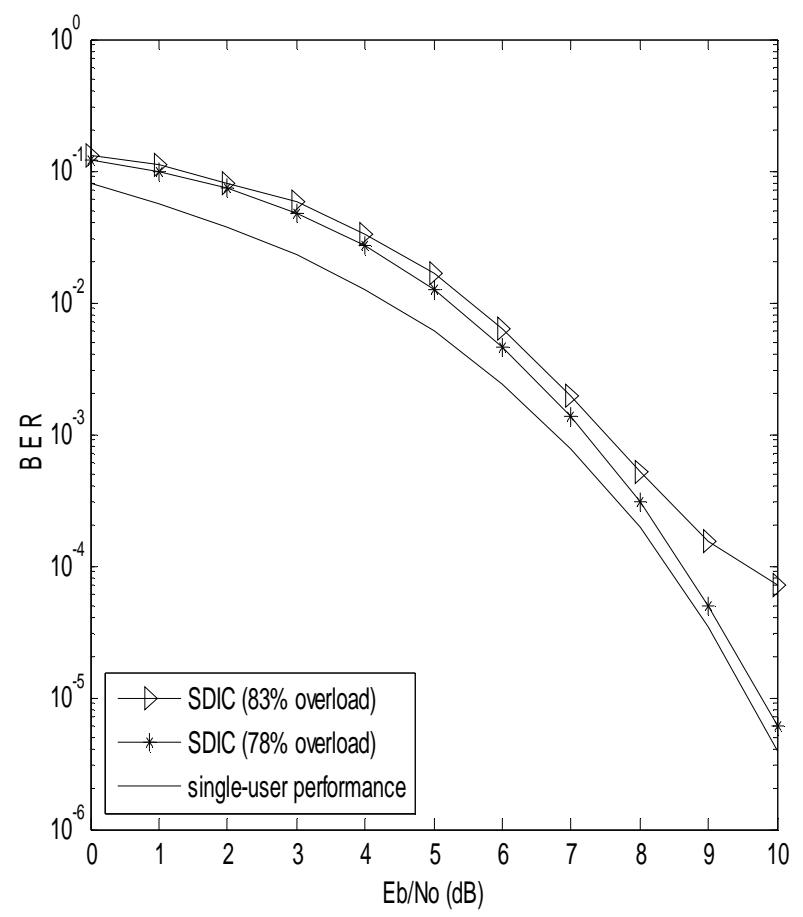

Figure 7. BER performance comparison of OG/OG scheme with periodic complex scrambling with Soft decision Interference cancellation (SDIC) for $\mathbf{N}=64$.

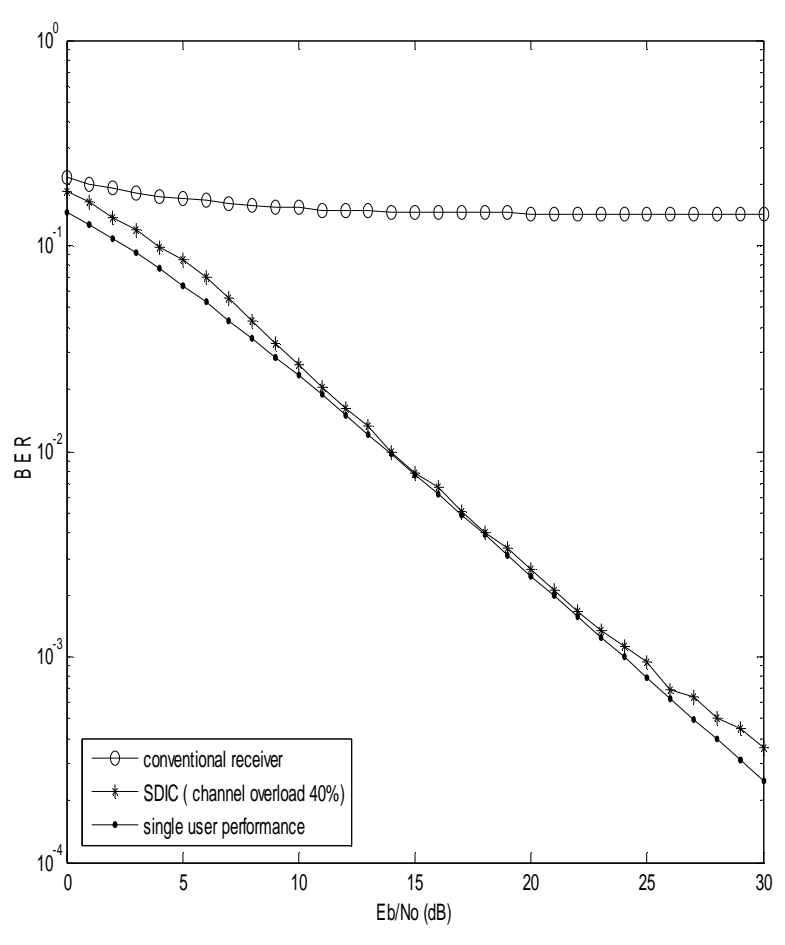

Figure 8. Mean BER performance of OG/OG with $40 \%$ overload and SDIC receiver over a Rayleigh fading channel without scrambling.

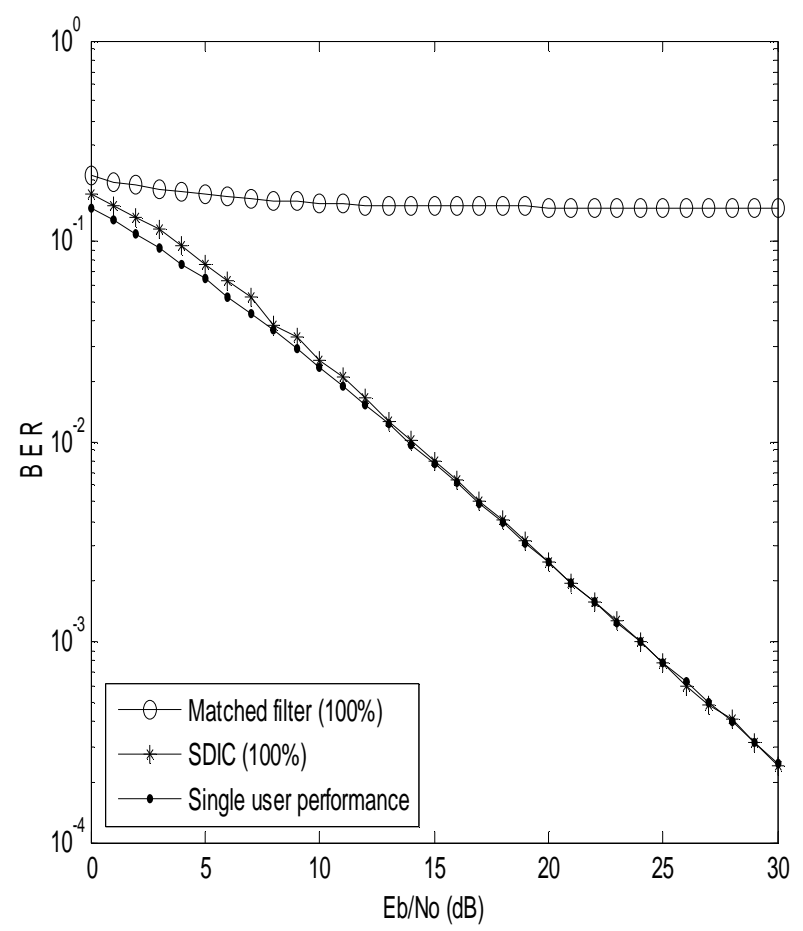

Figure 9. BER performance of s-OG/OG scheme with $100 \%$ overloading with random complex scrambling over a Rayleigh fading channel for $\mathrm{N}=64$. 
[3] S. Verdu, "Multi-user detection," Cambridge University Press, 1998.

[4] J. A. F. Ross and D. P. Taylor, "Vector assignment scheme for $\mathrm{M}+\mathrm{N}$ users in N-dimensional global additive channel," Electronics Letters, Vol. 28, August 1992.

[5] R. E. Learned, A. S. Willisky, and D. M. Boroson, "Low complexity joint detection for oversaturated multiple access communications," IEEE Transactions Signal Processing, Vol. 45, pp. 113-122, January 1997.

[6] F. Vanhaverbeke, M. Moeneclaey, and H. Sari, "DS/CDMA with two sets of orthogonal sequences and iterative - detection," IEEE Communications Letters, Vol. 4, pp. 289-291, September 2000.

[7] F. Vanhaverbeke and M. Moeneclaey, "Critical load of oversaturated systems with multistage successive interference cancellation," IEEE VTC, Vol. 4, pp. 26632666, April 2003.

[8] D. Djonin and V. K. Bhargava, "New results on low complexity detectors for oversaturated CDMA systems," in Proceedings of Globecom 2001, pp. 846-850, November 2001.

[9] P. Kumar and S. Chakrabarti, "A new overloading scheme for DS-CDMA system," National Conference on Communication, IIT Kanpur, pp. 285-288, 26-28 January 2007.

[10] K. Yang, Y. K. Kim, and P. V. Kumar, "Quasi-orthogonal sequences for code-division multiple-access systems," IEEE Transactions on Information Theory, Vol. 46, pp. 982-993, May 2000.

[11] Physical Layer Standard for cdma2000 Spread Spectrum Systems, Realse B, TIA/EIA 3GPP2 C.S0002-B, January 16, 2001.

[12] H. Donelen and T. O. Farrell, "Methods for generating sets of orthogonal sequences," Electronics Letters, Vol. 35, pp. 1537-1538, September 1999.

[13] P. Kumar and S. Chakrabarti, "A new overloading scheme for cellular DS-CDMA using orthogonal gold codes," IEEE Vehicular Technology Conference (VTC), pp. 10421046, May 2008. 